\title{
Crack propagation modeling using Peridynamic theory
}

\author{
M.H.Hafezi ${ }^{\mathrm{a}}$, R. Alebrahim ${ }^{\mathrm{b}}$, and T. Kundu ${ }^{\mathrm{a}}$ \\ ${ }^{a}$ Department of Civil Engineering and Engineering Mechancis, University of Arizona Tucson, \\ AZ 85721, USA \\ ${ }^{\text {b}}$ Department of Mechanical and Materials Engineering, Universiti Kebangsaan Malaysia 3600 \\ bangi Selangor, D.E Malaysia
}

\begin{abstract}
Crack propagation and branching are modeled using nonlocal peridynamic theory. One major advantage of this nonlocal theory based analysis tool is the unifying approach towards material behavior modeling - irrespective of whether the crack is formed in the material or not. No separate damage law is needed for crack initiation and propagation. This theory overcomes the weaknesses of existing continuum mechanics based numerical tools (e.g. FEM, XFEM etc.) for identifying fracture modes and does not require any simplifying assumptions. Cracks grow autonomously and not necessarily along a prescribed path. However, in some special situations such as in case of ductile fracture, the damage evolution and failure depend on parameters characterizing the local stress state instead of peridynamic damage modeling technique developed for brittle fracture. For brittle fracture modeling the bond is simply broken when the failure criterion is satisfied. This simulation helps us to design more reliable modeling tool for crack propagation and branching in both brittle and ductile materials. Peridynamic analysis has been found to be very demanding computationally, particularly for real-world structures (e.g. vehicles, aircrafts, etc.). It also requires a very expensive visualization process. The goal of this paper is to bring awareness to researchers the impact of this cutting-edge simulation tool for a better understanding of the cracked material response. A computer code has been developed to implement the peridynamic theory based modeling tool for two-dimensional analysis. A good agreement between our predictions and previously published results is observed. Some interesting new results that have not been reported earlier by others are also obtained and presented in this paper. The final objective of this investigation is to increase the mechanics knowledge of self-similar and self-affine cracks.
\end{abstract}

Keywords: crack branching, Peridynamic theory, damage, thin plate

\section{INTRODUCTION}

The primary goal of continuum mechanics of deformable solids is to find displacement, strain and stress fields. It utilizes four fundamental conservation principles (balance laws) in mechanics, namely conservation of (i) mass (ii) linear momentum, (iii) angular momentum and (iv) energy to describe the material behavior under external loads. Of particular interest is the phenomenon of fracture. Newton (1643-1727), Euler (1707-1783), Lagrange (1736-1813) and Cauchy (1789-1857) had provided mathematical foundation of many physical phenomena. With the advancement of computers, scientists and engineers could model more complex material response more accurately. It brought more attention to the development of numerical techniques for existing analytical approaches (e.g. FEM, BEM, XFEM etc.). These methods systematically developed in the form of available commercial computational software like Abaqus, ANSYS, MATLAB, etc. Today, the majority of engineering design (e.g. vehicles, ships, aircrafts, trains, power plants, etc.) takes the benefit of these software for simulations, resulting big savings and better management of our environmental resources.

Many structural components of aircraft, ship and automobile consist of thin plates stiffened by ribs and stringers. ${ }^{1}$ For these and other applications of thin plates in structures the development of a simulation tool for thin plate analysis has significant importance. Classical (local) solid mechanics theory is not capable of predicting

Further author information: (Send correspondence to T.Kundu)

Tribikram Kundu.: E-mail: tkundu@email.arizona.edu, Telephone: 15206216573

Health Monitoring of Structural and Biological Systems 2016, edited by Tribikram Kundu,

Proc. of SPIE Vol. 9805, 98050P · C 2016 SPIE · CCC code: 0277-786X/16/\$18 · doi: 10.1117/12.2219487

Proc. of SPIE Vol. $980598050 \mathrm{P}-1$ 
out-of-plane deformation generated by in-plane loading in thin plates. The inconsistency must be attributed to the initial assumption, upon which the classical theory of plate is based. In other words, the classical theory is not entirely satisfactory for determining the transverse shears in plate. ${ }^{2}$ A peridynamic formulation is assessed here for a thin plate containing a crack identical to the problem geometry considered by Taylor and Steigmann. ${ }^{3}$

The word Peridynamic comes from the Greek word peri meaning near and dyna or force. This theory was first proposed by Silling. ${ }^{4}$ Peridynamic theory originally revised the classical theory of solid mechanics that uses partial differential equation and replaced it by integro-differential equation. Therefore, the continuum assumption that the material remains continuous after deformation is no longer needed. As a result discontinuities (e.g. cracks) can be handled. Peridynamic modeling also has the capability of capturing all failure modes while continuum mechanics based modeling approach often finds it difficult to predict crack paths by traditional simulation tool. This advantage of the technique attracted researchers to peridynamic theory for material behavior modeling and advancement of structural integrity analysis. It is also worth to design a Peridynamic based simulation tool for variety of problems in the field. Crack propagation and branching phenomena are illustrated through a few examples. Three final objectives of this study are $(i)$ To model crack propagation and branching, (ii) numerical implementation of peridynamic thin plate theory and (iii) study of out-of-plane deformation from in-plane loading that classical (local) theory is not capable of doing

\section{PERIDYNAMIC EQUATION OF MOTION}

There are two forms of peridynamic theory namely $(a)$ bond-based theory and $(b)$ state-based theory. ${ }^{5}$ Peridynamic equation of motion was introduced as an integro-differential equation in the following form. This equation yields the acceleration of collocation (material) points.

$$
\rho(\mathbf{x}) \ddot{\mathbf{u}}(\mathbf{x}, t)=\int_{\mathcal{H}} \mathbf{f}\left(\mathbf{u}\left(\mathbf{x}^{\prime}, t\right)-\mathbf{u}(\mathbf{x}, t), \mathbf{x}^{\prime}-\mathbf{x}\right) d V_{\mathbf{x}^{\prime}}+\mathbf{b}(\mathbf{x}, t)
$$

where $\mathbf{x}$ is the position vector in reference configuration of the body, $\rho$ is density which is constant, $\mathbf{u}$ is displacement vector, and $\mathbf{b}$ is a prescribed body force density vector. $\mathcal{H}$ is a neighborhood of radius $\delta$, where $\delta$ defines the size (radius) of the horizon for the material. Fig. 1 shows the peridynamic body and its motion. It also shows dual force density $\mathbf{f}$ between two particles that has contributions from the bond force density $\mathbf{t}$ at both particles. Note that $\mathbf{f}$ is antisymmetric. The function $\mathbf{f}$ is called the dual force density and is the basic quantity produced by peridynamic theory. It has dimensions of force per unit volume squared. The peridynamic equation of motion is an integral equation in space (left-hand side) and contains derivative term in time domain (right-hand side). This integro-differential equation, in essence is valid for every element of volume or surface and not just for infinitesimal volume or surface elements isolated from a body. This makes peridynamic theory distinct from the classical (local) theory. Hence, now the question is when nonlocality is important? One quick answer can be - if the deformed body contains cracks, then singularity appears at the crack tips, therefore differential terms (in equilibrium equation) cannot be defined at those points. Clearly, classical (local) theory has a weakness. So, nonlocality here is significant. Second question is how does the degree of nonlocality influence on simulation results? The degree of nonlocality has been suggested to be controlled by the size of horizon that makes peridynamic as a multi-scale modeling tool. However, there is still no physical interpretation of the horizon. 

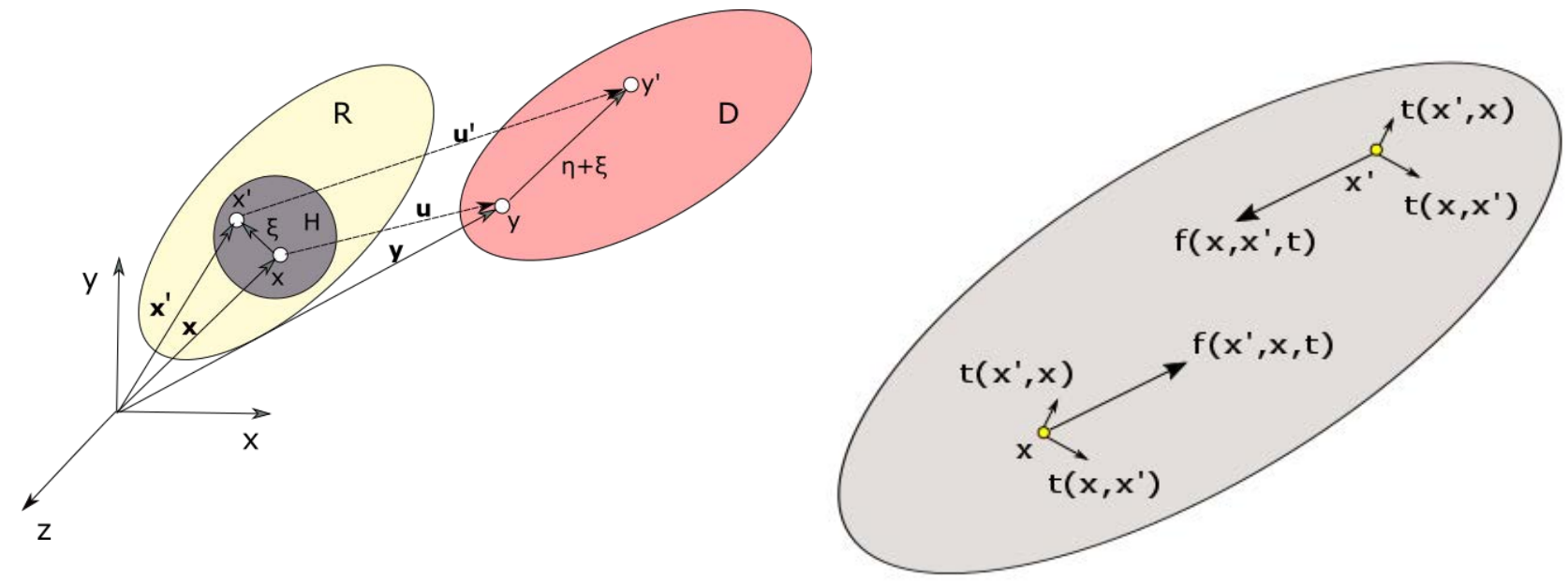

Figure 1. Deformation of a body: $R$ is the reference configuration and $D$ is the deformed configuration (left) and Dual force density $\mathbf{f}$ between two points has contributions from the bond force density $\mathbf{t}$ at both points (right)

\subsection{Peridynamic formulation}

Consider a mechanical component having one dimension significantly smaller than the other two dimensions, which is subjected to external time-dependent excitation forces acting in the plane defined by its two significant dimensions. Obviously, the significant displacements of the vibrating medium are lying on the same 2D plane. In this context, the governing equation of motion of any particle inside the vibrating 2D medium is given by the equation (1). The second-order derivatives of displacements $(u, v)$ with respect to time is denoted by $\ddot{\mathbf{u}}$. Position vectors are defined as $\mathbf{x}(x, y)$ and $\mathbf{x}^{\prime}\left(x^{\prime}, y^{\prime}\right)$. Note that $\rho(\mathbf{x})$ is the material density, and body force densities are given by vector $\mathbf{b}(\mathbf{x}, t)$ in $2 \mathrm{D}$ plane. $\mathbf{f}$ represents the force function measured in force per unit volume squared exerted on the point $\mathbf{x}$ by the point $\mathbf{x}^{\prime}$, and $\mathcal{H}$ is the domain of integration. In equation (1) $\mathbf{x}^{\prime}$ is restricted by the position of $\mathbf{x}$ by defining the relative position as

$$
\boldsymbol{\xi}=\mathbf{x}-\mathbf{x}^{\prime} \quad|\xi| \leq \delta
$$

The distance $\delta$ is called horizon which represents the distance of the nonlocal approximation. The domain $\mathcal{H}$ for every give material point $\mathbf{x}^{\prime}$ is defined by the equation

$$
\mathcal{H}\left(\mathbf{x}^{\prime}\right)=\mathbf{x}^{\prime}:|| \mathbf{x}-\mathbf{x}^{\prime} \mid \leq \delta
$$

Obviously, horizon in 2D plane is a circular disc of radius $\delta$ centered at $\mathbf{x}^{\prime}$. Another fundamental measure of peridynamic theory is the bond stretch given by

$$
s=\frac{|\boldsymbol{\xi}+\boldsymbol{\eta}|-|\boldsymbol{\xi}|}{|\boldsymbol{\xi}|}
$$

where $\eta$ is the relative displacement. Strictly speaking, it has been used to determine whether the bond has failed or not, thus it is the measure governing the force between the particles (e.g. similar to strain in classical theory). Bonds which exceed the predetermined value $s_{0}$ are considered as damaged. In linear peridynamics, the force amplitude is given by the equation

$$
|\mathbf{f}(\boldsymbol{\xi}, \boldsymbol{\eta})|=c \frac{|\boldsymbol{\eta}|}{|\boldsymbol{\xi}|}
$$


In the bond based theory the force vector is aligned with the vector $\boldsymbol{\eta}$, and for a $2 \mathrm{D}$ plate the bond constant is given by. ${ }^{3}$

$$
c=\frac{9 E}{2 \pi \delta^{3}}
$$

The kinematics of peridynamic expression can be compared against those balance laws in nonlocal and classical (local) theories (e.g. deformation gradient vs deformation state). More detail derivation has been shown by Silling and Lehoucq. ${ }^{5}$ Now, bond based peridynamic theory can be followed if as a special case the force density vectors $\mathbf{t}$ are equal in magnitude and parallel to the relative position vectors $\xi+\eta$ in deformed configuration. In the case when they do not hold equality but remain in parallel to the relative position vectors in deformed configuration, then it is called ordinary-state peridynamic theory. Note that only material points within horizon have interactions. As mentioned earlier, the physical meaning of horizon is not accurately specified yet. However, some detailed analysis has been reported by Bobaru and Zhang. ${ }^{6}$

\section{PERIDYNAMIC THEORY FOR THIN PLATES}

A two-dimensional bond-based peridynamic theory from three-dimensional bond-based peridynamic theory is proposed by Taylor and Steigmann. ${ }^{3}$ A brief summary of the two-dimensional plate model is given here. For a material point in the reference configuration, the position vector can be written as

$$
\mathbf{x}=\mathbf{v}+\zeta \mathbf{k}
$$

Similarly, the position vector in deformed configuration can be written as

$$
\mathbf{x}^{\prime}=\mathbf{v}^{\prime}+\zeta^{\prime} \mathbf{k}
$$

where $\mathbf{v}=(\mathbf{I}-\mathbf{k} \otimes \mathbf{k}) \mathbf{x}$ and $\zeta=\mathbf{k} \cdot \mathbf{x}$. Acceleration term in peridynamic equation of motion (1) has been approximated using Tylor series. Subtracting left hand side of Peridynamic equation for reference plane $(\zeta=0)$ and top surface $(\zeta=\epsilon)$ yields

$$
\rho\left(\epsilon \ddot{\mathbf{a}}+\frac{1}{2} \epsilon^{2} \ddot{\mathbf{b}}+\frac{1}{6} \epsilon^{3} \ddot{\mathbf{c}}+O\left(\epsilon^{3}\right)\right)=\int_{\mathcal{R}} \int_{0}^{\epsilon}\left[\mathbf{f}\left(\eta_{\epsilon}, \xi_{\epsilon}\right)-\mathbf{f}\left(\eta_{0}, \xi_{0}\right)\right] d \zeta^{\prime} d A^{\prime}
$$

where

$$
\begin{aligned}
\mathbf{a}(\mathbf{v}, t) & =\frac{\partial \mathbf{u}_{\mathbf{0}}(\mathbf{v}, t)}{\partial \zeta} \\
\mathbf{b}(\mathbf{v}, t) & =\frac{\partial^{2} \mathbf{u}_{\mathbf{0}}(\mathbf{v}, t)}{\partial \zeta^{2}} \\
\mathbf{c}(\mathbf{v}, t) & =\frac{\partial^{3} \mathbf{u}_{\mathbf{0}}(\mathbf{v}, t)}{\partial \zeta^{3}}
\end{aligned}
$$

A coupled system for $\left(\mathbf{u}_{\mathbf{0}}(v, t), \mathbf{a}(\mathbf{v}, t)\right)$ was derived that governed the particle motion on the reference surface.

$$
\begin{aligned}
\rho \epsilon \ddot{\mathbf{u}}_{0}(\mathbf{v}, t)= & \epsilon \int_{\mathcal{R}} \mathbf{f}\left(\eta_{00}, \xi_{00}\right) d A^{\prime} \\
& +\frac{1}{2} \epsilon^{2} \int_{\mathcal{R}}\left(\left[\frac{\partial \mathbf{f}}{\partial \eta}\left(\eta_{00}, \xi_{00}\right)\right] \mathbf{a}^{\prime}(\mathbf{v}, t)+\left[\frac{\partial \mathbf{f}}{\partial \xi}\left(\eta_{00}, \xi_{00}\right)\right] \mathbf{k}\right) d A^{\prime} \\
\rho \epsilon \ddot{\mathbf{a}}=\epsilon^{2} & {\left[\int_{\mathcal{R}}\left[\frac{\partial \mathbf{f}}{\partial \eta}\left(\eta_{00}, \xi_{00}\right)\right] d A^{\prime}\right] \mathbf{a}-\epsilon^{2}\left[\int_{\mathcal{R}}\left[\frac{\partial \mathbf{f}}{\partial \xi}\left(\eta_{00}, \xi_{00}\right) d A^{\prime}\right]\right] \mathbf{k} }
\end{aligned}
$$

These two coupled equations (11a) and (11b) are solved for $\left\{\left(\mathbf{u}_{\mathbf{0}}(v, t), \mathbf{a}(\mathbf{v}, t)\right\}\right.$. 


\section{NUMERICAL EXAMPLES}

Numerical examples are presented to verify the proposed peridynamic theory for a thin plate, the problem geometry analyzed is identical to the geometry considered by Tylor and Steigmann. ${ }^{3}$ In these examples, a thin cracked plate subjected to tensile load is studied. One plate contains a horizontal crack and the second plate contains an inclined crack at the center. Simple static loading conditions are considered first to demonstrate peridynamic predictions for the deformation and fracture of a plate with a central crack. It illustrates the capability of the proposed peridynamic formulation for thin plates. A square plate of dimension $50 \mathrm{~mm}$ by 50 $\mathrm{mm}$ and thickness $0.1 \mathrm{~mm}$ contains a crack at the center.In the first case geometry, the crack is oriented parallel to the edges where the loading is applied. In the second case geometry the crack is oriented at $45^{\circ}$ with respect to all edges. Elastic moduli, solid mass densities, and numerical load parameters are same as those given by Tylor and Steigmann. ${ }^{3}$

\section{RESULTS AND DISCUSSION}

The damage in the plate at various times is shown in Fig. 2. One can see that the crack initially extends horizontally along its original axis in a self-similar manner followed by branches. Our analysis has shown that branches spontaneously appear around $t=6 \mu \mathrm{s}$. Thereafter, more crack branches are formed. This type of branching is similar to what has been predicted by Taylor and Steigmann ${ }^{3}$ and Ha and Bobaru. ${ }^{7}$ However, interestingly the formation of damaged regions between crack tips and vertical edges are different from that predicted in Ha and Bobaru. ${ }^{7}$ Since the out-of-plane deformation was not considered in Ha and Bobaru ${ }^{7}$ a very sharp crack branch was reported. For the sake of brevity results without out-of-plane are not reported here. We observed that the intensity of damaged regions between crack tip and vertical edges is different from the results reported by Taylor and Steigmann. ${ }^{3}$ Fig. 3 shows results for the x-displacements and y-displacements; in both cases time as equal to $9 \mu \mathrm{s}$. It is also interesting to note that out of plane motion that begins at the boundaries where velocity field is applied moves as elastic waves towards the center. These are shown for horizontal central crack and inclined crack of Fig. 4. The results are symmetric and that is due to using a lateral surface as the reference surface. A region of broken nodes near the loaded boundaries is observed. 

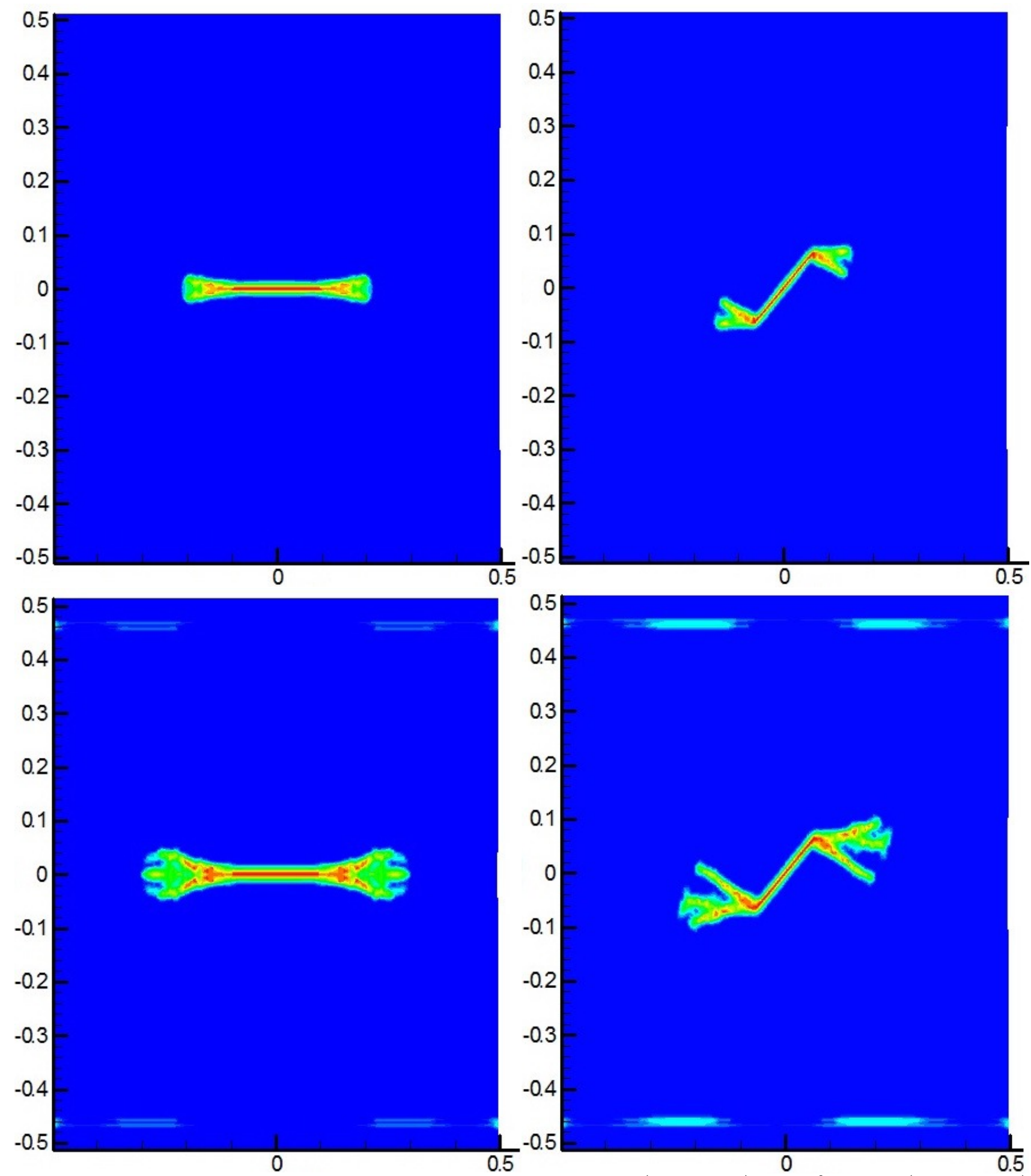

Figure 2. Contour plot of damage progression for a plate with horizontal (left column) and $45^{\circ}$ inclined (right column) central crack at $t=7.5 \mu s$ (top row), $t=9 \mu s$ (bottom row) 

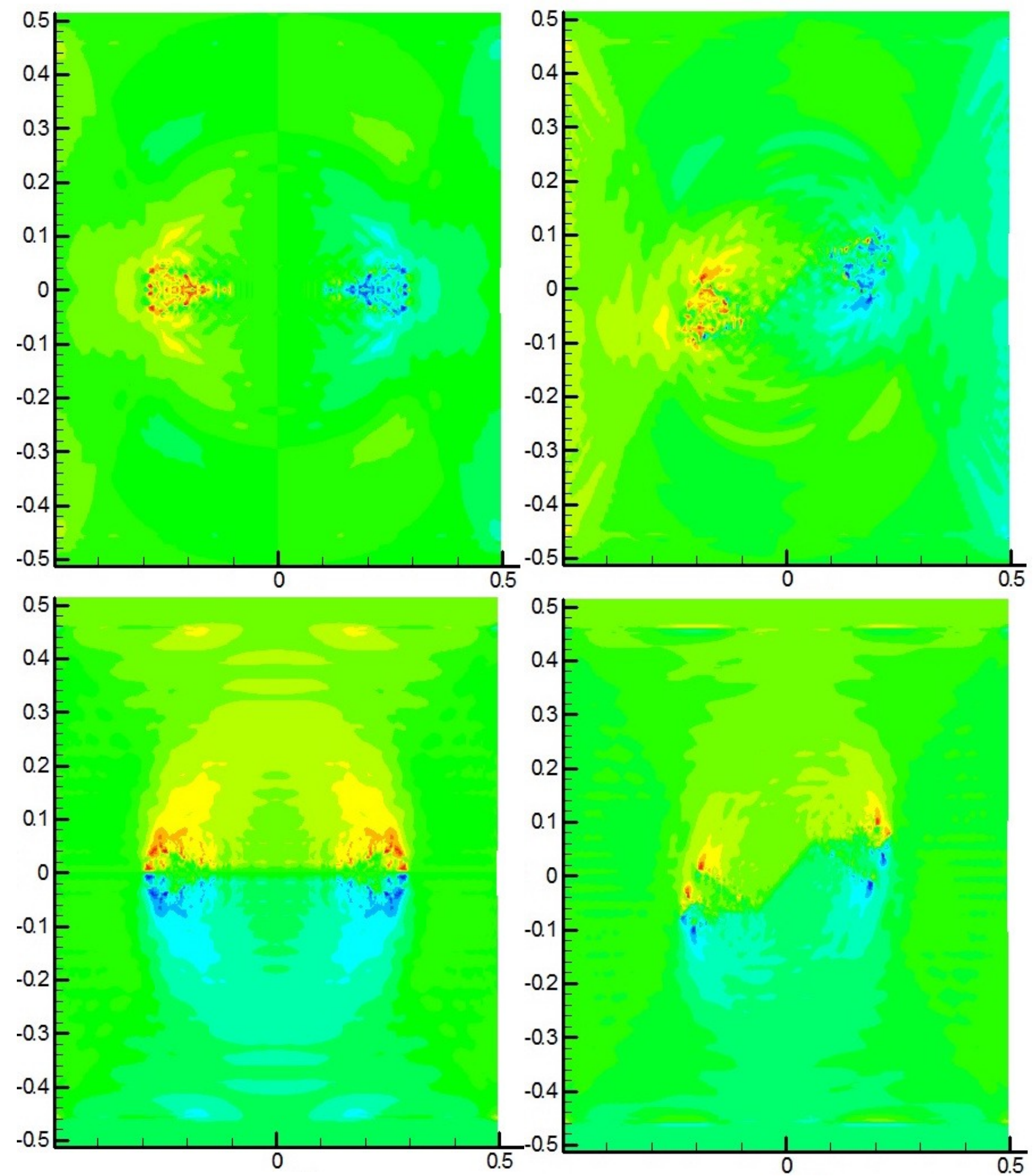

Figure 3. Contour plot of $\mathrm{x}$-displacement (top) and y-displacement (bottom) for a plate with a horizontal (left column) and a $45^{\circ}$ inclined (right column) central crack at $t=9 \mu \mathrm{s}$

Proc. of SPIE Vol. $980598050 \mathrm{P}-7$ 

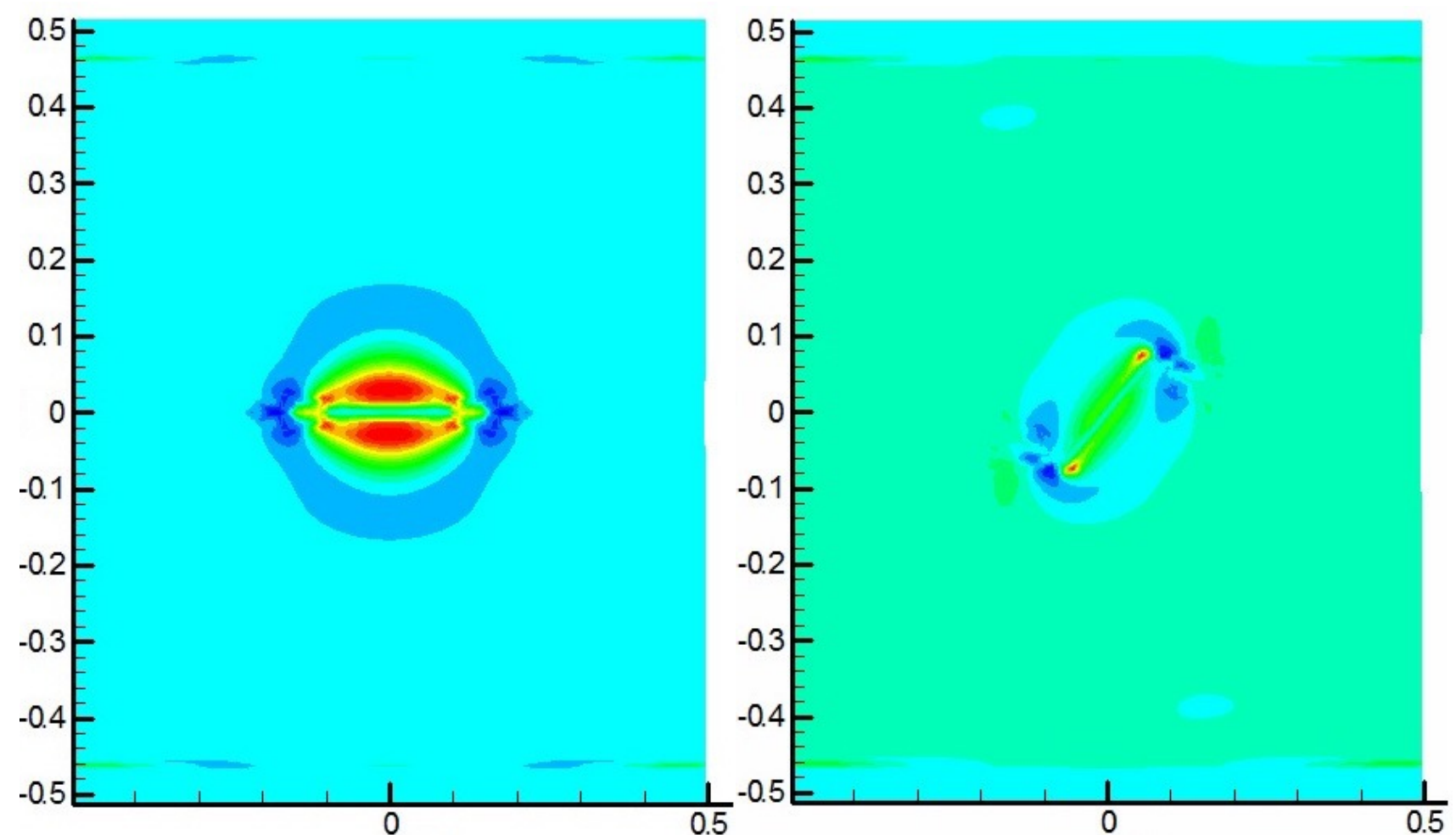

Figure 4. Contour plot of out-of-plane displacement for a plate with horizontal central (left) and inclined central crack (right) at $t=9 \mu s$

\section{CONCLUSIONS}

A two-dimensional peridynamic model for thin plates based on asymptotic analysis ${ }^{3}$ has been assessed. Selfsimilarity is observed and crack branching is correctly modeled. One major contribution of this work is to be able to model the out-of-plane deformation that classical (local) theory can not do. Parallel programming and high speed computation have been exercised. Future research will involve developing codes for more complex geometries that can be idealized as combinations of thin plates.

\section{ACKNOWLEDGMENTS}

The first author had a valuable discussion on high performance computing with Dr. Mark Borgstrom, in UITS at the University of Arizona.

\section{REFERENCES}

[1] Schijve, J., [Fatigue of Structures and Materials], Springer Netherlands (2009).

[2] Langhaar, H. L., [Energy Methods in Applied Mechanics], Wiley New York (1989).

[3] Taylor, M. and Steigmann, D. J., "A two-dimensional peridynamic model for thin plates," Mathematics and Mechanics of Solids 20(8), 998-1010 (2015).

[4] Silling, S., "Reformulation of elasticity theory for discontinuities and long-range forces," Journal of the Mechanics and Physics of Solids 48(1), 175 - 209 (2000).

[5] Silling, S. and Lehoucq, R., "Peridynamic theory of solid mechanics," in [Advances in Applied Mechanics], Aref, H. and van der Giessen, E., eds., Advances in Applied Mechanics 44, 73 - 168, Elsevier (2010).

[6] Bobaru, F. and Zhang, G., "Why do cracks branch? a peridynamic investigation of dynamic brittle fracture," International Journal of Fracture 196(1), 59-98 (2016).

[7] Ha, Y. D. and Bobaru, F., "Studies of dynamic crack propagation and crack branching with peridynamics," International Journal of Fracture 162(1), 229-244 (2010). 